\title{
Xenogeneic Tyrosinase DNA Vaccine
}

National Cancer Institute

\section{Source}

National Cancer Institute. Xenogeneic Tyrosinase DNA Vaccine. NCI Thesaurus. Code C68839.

A plasmid DNA vaccine, encoding an epitope of mouse tyrosinase, with potential antineoplastic activity. Administered via intramuscular electroporation, vaccination with xenogeneic tyrosinase DNA vaccine may induce both humoral and cytotoxic lymphocyte $(\mathrm{CTL})$ immune responses against melanoma cells that express tyrosinase, resulting in decreased tumor growth. 